\title{
Determination of the thermal offset of the Eppley precision spectral pyranometer
}

\author{
Martial Haeffelin, Seiji Kato, Amie M. Smith, C. Ken Rutledge, Thomas P. Charlock, \\ and J. Robert Mahan
}

\begin{abstract}
Eppley's precision spectral pyranometer (PSP) is used in networks around the world to measure downwelling diffuse and global solar irradiance at the surface of the Earth. In recent years several studies have shown significant discrepancy between irradiances measured by pyranometers and those computed by atmospheric radiative transfer models. Pyranometer measurements have been questioned because observed diffuse irradiances sometimes are below theoretical minimum values for a pure molecular atmosphere, and at night the instruments often produce nonzero signals ranging between +5 and -10 $\mathrm{W} \mathrm{m} \mathrm{m}^{-2}$. We install thermistor sondes in the body of a PSP as well as on its inner dome to monitor the temperature gradients within the instrument, and we operate a pyrgeometer (PIR) instrument side by side with the PSP. We derive a relationship between the PSP output and thermal radiative exchange by the dome and the detector and a relationship between the PSP output and the PIR thermopile output (net-IR). We determine the true PSP offset by quickly capping the instrument at set time intervals. For a ventilated and shaded PSP, the thermal offset can reach $-15 \mathrm{~W} \mathrm{~m}^{-2}$ under clear skies, whereas it remains close to zero for low overcast clouds. We estimate the PSP thermal offset by two methods: (1) using the PSP temperatures and (2) using the PIR net-IR signal. The offset computed from the PSP temperatures yields a reliable estimate of the true offset $\left( \pm 1 \mathrm{~W} \mathrm{~m}^{-2}\right)$. The offset computed from net-IR is consistent with the true offset at night and under overcast skies but predicts only part of the true range under clear skies. (C) 2001 Optical Society of America
\end{abstract}

OCIS codes: $120.0120,120.6810$.

\section{Introduction}

Pyranometers of varied manufacture are used in extensive networks around the globe to monitor solar radiation, such as the Atmospheric Radiation Measurement Program from the U.S. Department of Energy, the Baseline Surface Radiation Network of the World Climate Research Program, and the National Oceanic and Atmospheric Administration's surface radiation network SURFRAD. Shaded and unshaded instruments are used side by side to measure the diffuse and the global horizontal solar irradiances, respectively. The major uncertainties in

M. Haeffelin (m.p.haeffelin@larc.nasa.gov), A. M. Smith, and J. R. Mahan are with Virginia Polytechnic Institute and State University, Blacksburg, Virginia 24061. S. Kato is with Hampton University, Hampton, Virginia 23668. K. Rutledge is with Analytical Science and Materials, Hampton, Virginia 23666. T. Charlock is with NASA Langley Research Center, Hampton, Virginia 23681.

Received 11 May 2000; revised manuscript received 2 October 2000.

0003-6935/01/040472-13\$15.00/0

(C) 2001 Optical Society of America irradiances measured by pyranometers are due to the following limitations: (1) uncertainty in the absolute calibration of the instrument inherent in the calibration procedure, (2) uncertainty in angular response of the instrument and its application to the measured radiance field, and (3) detector sensitivity to IR radiation exchange within the instrument, often referred to as instrument offset. The errors associated with these limitations must be addressed quantitatively to reduce the measurement uncertainties.

Precise guidelines describe calibration procedures that ensure reliable and consistent derivation of instrument responsivity. ${ }^{1,2}$ Manufacturers attempt to characterize the directional response of pyranometers. They reveal deviations from a true cosine response of up to $10 \%$ at low solar elevations (zenith angle greater than $70^{\circ}$ ) that affect mainly pyranometer measurements of global downwelling solar irradiance. A recent study by $\operatorname{Reda}^{3}$ describes how to derive a directional dependence function from calibration data and how to apply this function to correct pyranometer measurements. Also, Michalsky et al. ${ }^{4}$ showed that the directional response problem can be greatly alleviated by measuring the global down- 
welling solar irradiance as the sum of the direct and diffuse components of downwelling solar irradiance.

The IR radiative exchange within the optical front end of a pyranometer causes a spurious output signal. This undesirable effect has been described extensively in the literature. ${ }^{5-7}$ In 1978 Gulbrandsen ${ }^{7}$ wrote that "in spite of its significance to pyranometer measurement, the zero point deviation and especially the depression, seems to have been more or less overlooked by authors discussing pyranometer data in recent year." Gulbrandsen ${ }^{7}$ estimates the zero point deviation that is due to heating or cooling of the pyranometer glass hemisphere to be up to $15 \%$ of a typical measured solar diffuse irradiance. He recommends either that the instrument body and dome temperatures be measured or that both the hot and the cold junctions of the thermopile be exposed to the IR radiation.

Because the instrument offset is driven by temperature gradients in the instrument, we refer to the offset in this paper as thermal offset. The thermal offset can be observed at night simply by monitoring of the pyranometer output signal. In the absence of any solar irradiance, the pyranometer output should be zero. Several data sets from field experiments (e.g., CAGEX, 8 the CERES-ARM GEWEX Experiment) reveal that the precision spectral pyranometer (PSP) output at night ranges from +5 to $-10 \mathrm{~W} \mathrm{~m}^{-2}$ depending on atmospheric and operating conditions. Some authors refer to this offset as the nighttime offset and assert that a similar offset must exist during daytime field measurements.

In the past five years several studies comparing solar irradiance measurements at the surface with theoretical atmospheric radiative transfer calculations have found discrepancies in the surface diffuse irradiance for clear skies. ${ }^{9,10}$ The models typically overestimate the diffuse solar irradiance at the surface by $10-30 \mathrm{~W} \mathrm{~m}^{-2}$. In addition, Cess ${ }^{11}$ reports that the measured diffuse irradiance is sometimes lower than the theoretical value for a pure molecular atmosphere. Reports of significant discrepancies between theory and measurements for clear skies are not, however, universal. ${ }^{12,13}$

Bush et al..$^{14}$ characterize the thermal offset in PSP's by measuring the temperature of the outside glass dome and of the instrument body. Bush et al. ${ }^{14}$ derive a relationship between the measured temperature difference and the nighttime pyranometer signal. During daytime operation, the pyranometer temperatures are measured, and a daytime thermal offset is determined. Bush et al. ${ }^{14}$ report daytime thermal offsets ranging from 0 to $-20 \mathrm{~W} \mathrm{~m}^{-2}$ for unventilated PSP's under conditions ranging from overcast to clear skies.

The present work builds on the empirical results presented by Bush et al. ${ }^{14}$ We describe the physics of the pyranometer and its thermal offset and propose correction techniques for both past and future solar irradiance measurements based on our understanding of the instrument.

In Section 2 we discuss the physics driving the

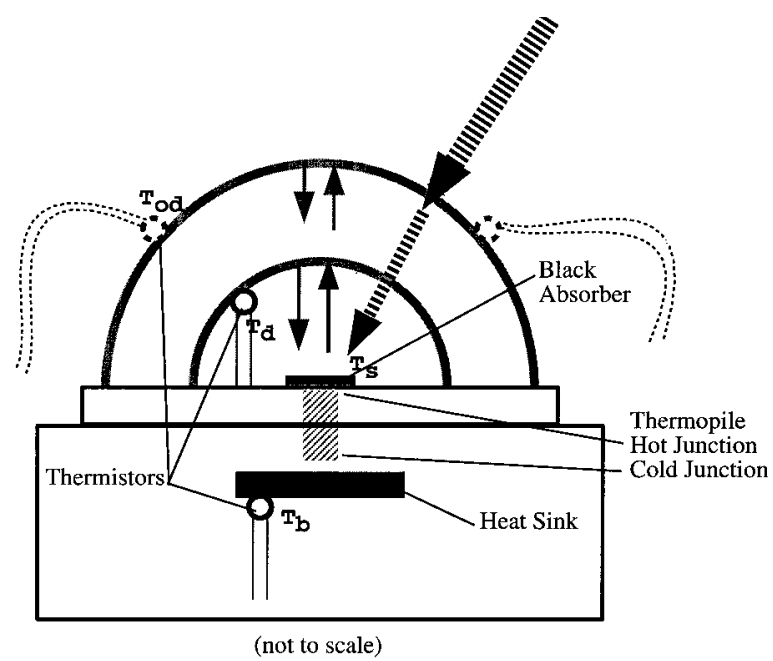

Fig. 1. Schematic depiction of a pyranometer with a thermistor bonded to the inner dome and a thermistor embedded in the heat sink next to the cold junction of the thermopile. The outer-dome thermistors, used in the laboratory experiment, are shown by dashed curves. The large black arrows represent incoming solar radiation, and the narrow gray arrows indicate the exchange of IR radiation between the domes and the detector.

Eppley PSP and the thermal offset as well as the modifications that allow us to measure temperature gradients within the instrument. In Section 3 we describe the laboratory experiments performed to establish a relationship between the temperature gradients and the thermal offset, and then we apply our technique to estimate and correct the thermal offset in daytime and nighttime field measurements. The results based on our modified PSP and the possibility of correcting past solar irradiance measurements are discussed in Section 4.

\section{Pyranometer and Its Thermal Offset}

\section{A. Description of a Precision Spectral Pyranometer}

A pyranometer is an instrument used to measure hemispherical broadband solar irradiance. As depicted in Fig. 1, solar radiation is transmitted through the two hemispherical glass domes and absorbed by a layer of Parsons' Black paint. The glass domes are predominantly transparent to solar radiation within the $0.3-2.5-\mu \mathrm{m}$ range but are considered to be opaque to longer wavelengths. The black absorber is intimately bonded to a thermopile detector to allow maximum heat transfer of the absorbed radiation. A thermopile detector is sensitive to both solar and IR radiation (0.3-100- $\mu \mathrm{m}$ spectral range). The thermopile consists of $\sim 40$ thermocouple junctions connected in series ${ }^{15}$; the hot junctions are exposed to the incident radiation, whereas the cold junctions face a heat sink. The temperature of the hot junctions is virtually the same as that of the black absorber. The cold junctions are maintained at the temperature of the heat sink by radiative heat transfer. The heat sink is in intimate thermal contact with the body of the instrument. In the absence of 
any temperature gradients in the instrument and any direct radiative input on the absorber, the hot junctions remain at the same temperature as the cold junctions, and the voltage output of the instrument should then be zero. When the temperatures of the hot junctions and cold junctions differ, the thermopile generates a voltage. This voltage is the output signal, which can be related to the radiative input through calibration.

The temperature of the black absorber is influenced by the net radiation at its surface. It absorbs the solar radiation transmitted through the domes as well as IR radiation emitted by the components of the instrument optical front end. The absorber also emits radiation proportionally to the fourth power of its temperature. The temperature of the absorber increases as the incident solar radiation increases, and so does the IR radiation emitted by the absorber. The corresponding net radiation absorbed is therefore less than the solar radiation increase. This effect is accounted for in calibration because the increased thermal irradiance emitted by the detector is proportional to the incoming solar radiation, provided the temperature increase of the detector does not exceed a few degrees. This effect is therefore not considered an offset, but can become an issue if the instrument is operated at a temperature significantly different from the calibration temperature, in which case the temperature dependence of the instrument sensitivity should be accounted for.

The absorber exchanges IR radiation with the inner dome and the shiny metal base of the dome. It also exchanges radiation with the outer dome in the spectral intervals where the inner dome is not completely opaque. The hemispherical emissivity integrated over the emission spectrum of a blackbody at $300 \mathrm{~K}$ for the 1 -mm inner dome is $\sim 0.8$, whereas it is greater than 0.9 for a $2-\mathrm{mm}$ dome. ${ }^{16}$ The hemispherical reflectivity is close to 0.1 ; hence a $2-\mathrm{mm}$ dome can be considered opaque in the IR, whereas a 1-mm dome can transmit a small fraction of the radiation emitted by the absorber and the outer dome. ${ }^{17}$ The hemispherical emissivity of the metal base at 300 $\mathrm{K}$ is less than 0.1 . Its contribution is not significant, because the base and the absorber can exchange radiation only through reflection on the dome. The net-IR irradiance at the surface of the black absorber affects the temperature of the hot junctions of the thermopile and hence modifies the voltage output. This voltage offset varies with time as a function of the thermal gradients in the instrument. Our goal is the determination of that offset.

\section{B. Thermal Offset in the Pyranometer}

The net (downwelling minus upwelling) IR irradiance at the surface of the Earth is typically negative; the surface loses IR radiation to the clear sky or to clouds. Both the body and the outer dome of the pyranometer are similarly cooled by the loss of IR radiation. If the body and the outer-dome temperatures differ, so will their respective losses of heat in the IR. Temperature differences between the outer dome and body of the instrument can be attributed to the following reasons: (1) The dome has a small heat capacity that allows it to change temperature quickly, (2) the dome has a small thermal conductivity that creates a temperature gradient between its tip and base, (3) the dome is directly facing the sky and has a hemispherical IR emissivity of 0.9 , (4) the dome is transparent to a large fraction of the solar spectrum $(0.3-2.5 \mu \mathrm{m})$, and (5) the instrument remains in a transient thermal state because of changes in surface and air temperatures, wind, and precipitation.

Variations in the convective heat transfer between the ambient air and the outer dome can be reduced by a ventilator system that blows a constant airflow on the surface of the outer dome. The ventilator can moderate the radiative cooling of the outer dome exposed to a cold sky and bring the outer-dome temperature closer to the instrument body temperature. The inner-dome temperature is coupled radiatively to the temperature of the outer dome. However, the temperature gradient between the inner dome and the body is significantly less than the temperature gradient between the outer dome and the body. Therefore the inner dome acts as an efficient, but not perfect, IR radiation shield between the detector and the outer dome.

The output voltage of a thermopile is proportional to the temperature difference between its hot and its cold junctions. The temperature of the inner dome can affect the temperature difference between the hot and the cold junctions of the thermopile and therefore modify the thermopile voltage output. In this paper we show that the thermal offset of the pyranometer can be related to the difference between the inner-dome temperature and the instrument body temperature, which is the nominal detector temperature (i.e., that of the cold junctions). Bush et al. ${ }^{14}$ established a relationship between the thermal offset and the outer-dome-tobody temperature difference. The two domes are coupled radiatively, so the two approaches are defendable. We choose to use the inner dome for the following reasons: (1) The effective temperature of the inner dome can be monitored with a single thermistor (see Subsection 2.D), (2) the relationship between the thermal offset and the inner-dome-tobody temperature difference holds even during large thermal shocks (see Subsection 3.A), and (3) the modified instrument is as rugged as a standard PSP, so it can be tested and operated in a large variety of environments (see Subsection 2.E).

It is important to note that the thermal offset is not eliminated by the calibration process. For example, the calibration procedure currently recommended by the Baseline Surface Radiation Network ${ }^{18}$ consists of a series of measurements with a shaded pyranometer, an unshaded pyranometer, and a previously calibrated normal-incidence pyrheliometer (NIP) or narrow-field-of-view cavity radiometer traceable to World Radiation Monitoring Center radiometric 
standards. The two pyranometers are calibrated based on a solar component summation:

$$
F^{\mathrm{Glo}}=F^{\mathrm{Dif}}+\mu F^{\mathrm{Dir}},
$$

where $F^{\text {Glo }}$ is the global solar irradiance measured by the unshaded pyranometer, $F^{\text {Dif }}$ is the diffuse solar irradiance measured by the shaded pyranometer, $F^{\text {Dir }}$ is the direct solar radiation measured by a NIP or cavity pointed toward the Sun, and $\mu$ is the cosine of the solar zenith angle. Both global and diffuse irradiance measurements are affected by thermal offsets, but not of equal magnitudes or signs. The thermal offset is believed to be minimal in a NIP and nonexistent in a cavity. Therefore this calibration technique does not remove the offset. In addition, the thermal offset varies considerably with operating conditions.

\section{Analytical Model of the Pyranometer}

We developed an analytical model of the energy balance of the pyranometer to relate instrument temperatures to instrument offsets. This model is inspired from analyses carried out for the pyrgeometer (PIR) instrument by Albrecht et al. ${ }^{19}$ Philipona et al. ${ }^{20}$ and Fairall et al. ${ }^{21}$ The temperature gradient through the thermopile is a function of the net radiation absorbed by the detector, $F_{\text {net }}=\left(T_{s}-\right.$ $\left.T_{b}\right) / c$, where $T_{s}$ and $T_{b}$ are the hot and the cold junction temperatures, respectively, and $c$ is the sensitivity. As depicted in Fig. $1, F_{\text {net }}$ can also be defined as

$$
F_{\text {net }}=\alpha_{s} \tau_{d} E+\alpha_{s} \varepsilon_{d} \sigma T_{d}^{4}+\alpha_{s} \varepsilon_{s} \rho_{d} \sigma T_{s}^{4}-\varepsilon_{s} \sigma T_{s}^{4},
$$

where $\alpha_{s}$ and $\varepsilon_{s}$ are the absorptivity and the emissivity of the sensor, respectively; $\tau_{d}$ is the transmissivity of the dome in the solar spectral domain; $\varepsilon_{d}$ and $\rho_{d}$ are the emissivity and the reflectivity of the dome, respectively; $T_{d}$ is the temperature of the dome; and $E$ is the incoming solar irradiance. A voltage $U_{e}$ proportional to the temperature gradient is generated. We solve for $E$ and rewrite Eq. (2) in terms of the measurable quantities $U_{e}, T_{b}$, and $T_{d}$ :

$$
\begin{aligned}
E= & U_{e}\left[\frac{S}{\alpha_{s} \tau_{d} c}+\frac{4 \sigma S}{\tau_{d}}\left(1-\varepsilon_{s} \rho_{d}\right) T_{b}^{3}\right]+\frac{\varepsilon_{d} \sigma}{\tau_{d}}\left(\varepsilon_{s} T_{b}^{4}-T_{d}^{4}\right) \\
& +\frac{\sigma}{\tau_{d}}\left(1-\alpha_{s}\right) T_{b}^{4}
\end{aligned}
$$

where $S$ is the Seebeck coefficient of the thermopile. The first term on the right-hand side of Eq. (3) contains two terms within the brackets that represent the proportionality between the thermopile output voltage and the downwelling solar irradiance. The first term in the brackets is the effect of solar irradiance, whereas the second term accounts for the radiative effect of the temperature increase of the hot junction that is due to absorption of solar irradiance, as described in Subsection 2.A. The second term modifies the slope between $E$ and $U_{e}$ by $\sim 2.5 \%$. Both terms are accounted for in calibration. The other two terms of Eq. (3) represent the amount of irradiance that must be accounted for to compensate for the IR radiative exchange that takes place between the inner dome and the detector. If the absorptivity of the detector is assumed to be $\alpha_{s}=1.0$ instead of 0.98, the last term vanishes, and Eq. (3) can be greatly simplified, whereas the total IR irradiance is modified by less than $0.5 \%$. Equation (3) can be parameterized as

$$
E=C_{1} U_{e}+C_{2} \sigma\left(T_{b}^{4}-T_{d}^{4}\right),
$$

where $C_{1}$ is the instrument calibration coefficient and $C_{2}$ accounts for the optical and the radiative properties of the domes and the detector surface.

\section{Dome Temperature Measurements}

We use small thermistors to measure the temperatures of the inner dome and the instrument body to determine the thermal offset of the PSP. The temperature of a glass dome exposed to solar radiation is difficult to measure because the solar radiation $a b-$ sorbed by the dome and the attached thermistor can differ significantly. A standard Yellow Springs Instruments (YSI) 44031 thermistor has a 1-2-mm head with a black coating. Such a thermistor can warm up several degrees when exposed to solar radiation compared with a shaded condition. ${ }^{22}$ Experiments described by Haeffelin et al. ${ }^{22}$ show that measurement of the glass dome temperature can be made more representative by reducing the crosssectional area of the thermistor that is exposed to solar radiation, by coating the thermistor with a glossy white paint, by improving the bonding of the thermistor to the glass, and by shading the thermistor from direct solar radiation.

The domes, which are relatively poor thermal conductors, are generally not isothermal. To minimize the occulted fraction of diffuse irradiance, we measure the temperature of the inner dome with a single thermistor. The location of the thermistor is chosen to be representative of the temperature for the entire dome. This location is determined with an engineering finite-element analysis. A finite-element model was developed to represent the conductive heat transfer inside a simplified instrument composed of a metal body and a glass dome. ${ }^{23}$ The model simulates an instrument in an environment maintained at $273 \mathrm{~K}$ and exposed to a clear sky of brightness temperature $220 \mathrm{~K}$; it computes the temperature distribution on the dome. The IR irradiance emitted by the dome and incident upon the detector is determined from this temperature distribution. The effective temperature of the dome is the temperature of an isothermal dome that would produce the same irradiance. The optimal location for the thermistor is where it can measure a temperature equal to the effective temperature. This location is determined from the computed temperature distribution. Model tests include both transient and steady-state cases. As shown in Fig. 2, on average, the temperature at a $40^{\circ}$ elevation off the base is within $0.05{ }^{\circ} \mathrm{C}$ of the 


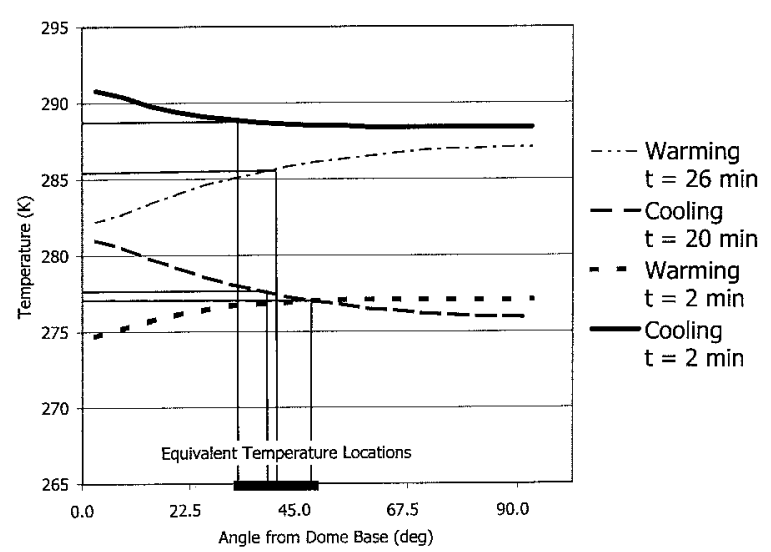

Fig. 2. One-dimensional temperature gradient along the inner dome at two different time steps of a cooling and a warming experiment. The optimal location for the thermistor to measure the effective temperature of the inner dome is shown by the vertical lines.

effective temperature. To prevent the dome thermistor from shading the direct solar beam to the detector surface, we place the thermistor on the dome at the azimuth $360^{\circ}$ (North). As for the diffuse solar radiation, the solid angle subtended by a 1 -mm thermistor is of the order of only $0.1 \%$ of the hemisphere. Hence the impact of the thermistor shadow on the measurement of the diffuse irradiance is negligible.

\section{E. Modified Precision Spectral Pyranometer}

We install a standard YSI 44031 thermistor in the heat sink of an Eppley PSP to monitor the temperature of the cold junctions. We bond a white reduced-size thermistor (YSI SP20796) onto the inside surface of the inner dome by using a thermally conductive white silicon paste. The inner-dome thermistor is installed at a $40^{\circ}$ elevation off the base and aligned azimuthally with the pin connector. We replace the four-pin connector of the standard PSP with a ten-pin connector to accommodate the thermopile and thermistor output. For the laboratory experiment, described in Subsection 3.A, two additional YSI 44031 thermistors are bonded to the outside surface of the outer dome. The thermistors are located $\sim 45^{\circ}$ off the base, one aligned azimuthally with the pin connector and the other on the opposite side. The locations of the thermistors are shown in Fig. 1. The outer-dome thermistors are removed for the field measurements described in Subsection 3.B.

The standard YSI 44031 thermistors are calibrated before installation. The thermistor are held in a bath whose temperature is controlled to better than $0.005{ }^{\circ} \mathrm{C}$. The outputs are monitored as the bath temperature is stepped through ten levels of temperatures between 0 and $50{ }^{\circ} \mathrm{C}$. The thermistor data are used to determine new coefficients for the Hart/ Steinhart equation that relates thermistor resistance to temperature. Ideally this allows us to measure the heat sink temperature with a $\pm 0.005{ }^{\circ} \mathrm{C}$ uncer-

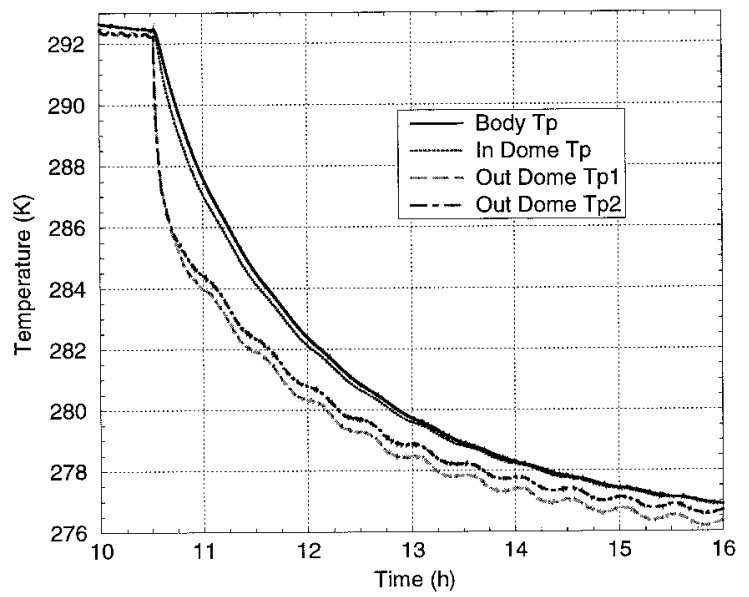

Fig. 3. Time series of detector temperature (solid curve), innerdome temperature (dotted curve), and two outer dome temperatures (dashed and dotted-dashed curves) during a cooling experiment.

tainty. We calibrate the YSI SP20796 thermistor bonded on the inner dome by maintaining the instrument in an isothermal chamber and matching its temperature to the heat-sink temperature.

\section{Experimental Determination of the Offset}

\section{A. Laboratory Simulation of Nighttime Measurements}

Our purpose in the laboratory experiment is to establish the relationship between the temperature gradients in the instrument (i.e., difference of detector and dome temperature) and the thermal offset. We perform the laboratory measurements in a dark room, so the instrument voltage output is due solely to the temperature gradients in the instrument.

A nonzero voltage output can be produced by a quick change in the temperature surrounding the PSP. We do this by moving the PSP in and out of a refrigerator. The domes and the body have different thermophysical properties and hence different time constants. The temperature of the environment is modified suddenly, creating a temperature gradient in the instrument and ultimately a thermopile voltage output.

Initially the modified PSP is left in a thermally stable environment for more than $2 \mathrm{~h}$ to minimize the temperature gradient through the instrument. During a cooling experiment, the PSP is placed in a refrigerator, which is approximately $20^{\circ} \mathrm{C}$ cooler than the laboratory, where it remains for almost $5 \mathrm{~h}$. A warming experiment consists in removing the PSP from the refrigerator and exposing it to the warmer air inside a box at the ambient temperature of the laboratory. The instrument is not ventilated in either experiment.

The temperatures of the four thermistors installed in our modified PSP are shown in Fig. 3 for a cooling experiment. Results of the warming experiments are not shown here because they do not provide additional information. Figure 3 shows a substantial drop in temperature with a significant lead time for 


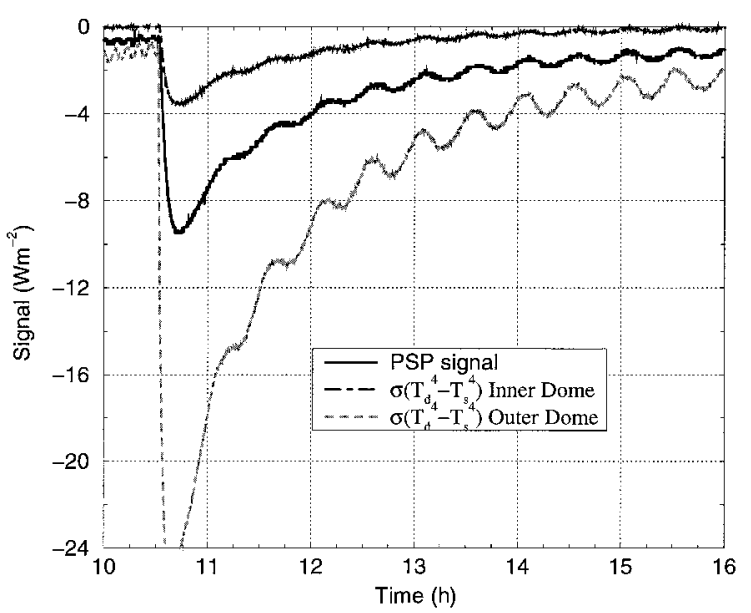

Fig. 4. Time series of the PSP output signal (solid curve), the theoretical blackbody radiative exchange between the inner dome and the detector (dotted-dashed curve), and between the outer dome and the detector (dashed line) during a cooling experiment.

the two thermistors on the outer dome. The temperature of the outer dome drops by $\sim 7^{\circ} \mathrm{C}$ in the first $5 \mathrm{~min}$, but it takes almost $5 \mathrm{~h}$ for the system to approach uniform temperature. The 20 -min oscillations of the outer-dome temperatures are due to the compressor cycles of the refrigerator. The difference between the two outer-dome temperatures is due to the nonuniform temperature distribution inside the refrigerator, which is in turn due to the location of a freezer compartment.

The theoretical blackbody radiative exchange between two elements of the instrument is given by $\sigma\left(T_{d}^{4}-T_{b}^{4}\right)$, where $T_{d}$ is the dome temperature and $T_{b}$ stands for the body temperature measured in the heat sink, which is the nominal detector temperature. Figure 4 shows the PSP output signal and the blackbody radiative exchange between the detector and the inner dome and between the detector and the outer dome, based on measured temperatures, for the cooling experiment. The blackbody exchange between the outer dome and the detector assumes no inner dome. The PSP output signal reaches a maximum $\sim 10 \mathrm{~min}$ after its insertion into the refrigerator. The blackbody radiative exchange between the detector and the outer dome predicted from their temperatures is approximately three times larger than the radiative exchange measured by the PSP. The temperature difference between the detector and the inner domes predicts a much smaller radiative exchange. The radiative exchange based on the inner-dome temperature is in phase with the PSP output, whereas that from the outer dome is shifted by several minutes. This is particularly noticeable in the case of a rapid temperature change.

Figure 5 shows the relationship between the theoretical inner-dome-to-detector radiative exchange and the PSP output signal during a cooling experiment. The data are fitted with a linear regression,

$$
V_{\text {Offset }}=A \sigma\left(T_{d}^{4}-T_{b}^{4}\right)+B .
$$

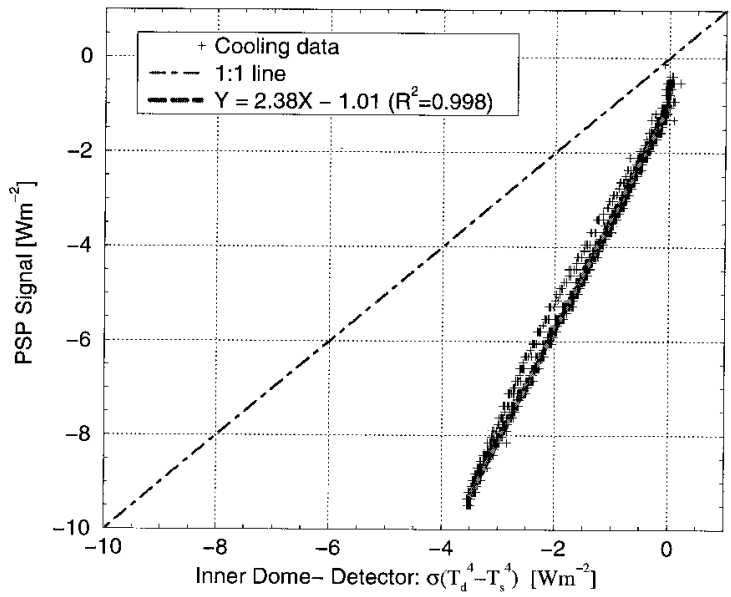

Fig. 5. Relationship between the inner-dome-to-detector theoretical blackbody radiative exchange and the PSP output signal during a cooling experiment.

The maximum error of the regression is less than 0.5 $\mathrm{W} \mathrm{m} \mathrm{m}^{-2}$. The variations in radiative exchange between the inner dome and the detector can explain over $99.8 \%$ of the PSP output signal. If the inner dome and the detectors were blackbodies and the radiative exchange between them were the only source of thermal offset, the slope of the regression would be 1 . The slope $A$ of 2.38 implies that the detector must exchange energy with another component or through another mode of heat transfer, because the detector loses more energy than predicted by the theoretical blackbody radiative exchange between the detector and the inner dome. Convective heat transfer could be significant when the detector is warmer than the dome, but not when the detector is colder than the dome. Convective transfer would produce different slopes for the cooling and the warming experiments, which is not the behavior observed in our experiments. We could interpret the slope of 2.38 as a radiative exchange between the detector and an element at a lower temperature than that of the inner dome. Because the outer dome is colder than the inner dome in the cooling experiment, a nonzero IR transmissivity of the 1-mm inner dome, consistent with its 0.8 IR emissivity described in Subsection 2.B, could explain the regression slope greater than 1 . The regression intercept of $1 \mathrm{~W} \mathrm{~m}^{-2}$ reveals a bias in our temperature measurements; the temperature gradient between the inner dome and the detector is overestimated by $0.05^{\circ} \mathrm{C}$.

Figure 6 shows the relationship between the theoretical outer-dome-to-detector radiative exchange and the PSP output signal during a cooling experiment. The data collected after the maximum PSP output can be fitted with a linear regression. The data that deviate significantly from the linear behavior were collected during the first $10 \mathrm{~min}$ of the experiment. This implies that the outer dome is not the primary direct influence on the detector temperature, i.e., most of the IR radiation emitted by the detector toward the outer dome is absorbed by the 


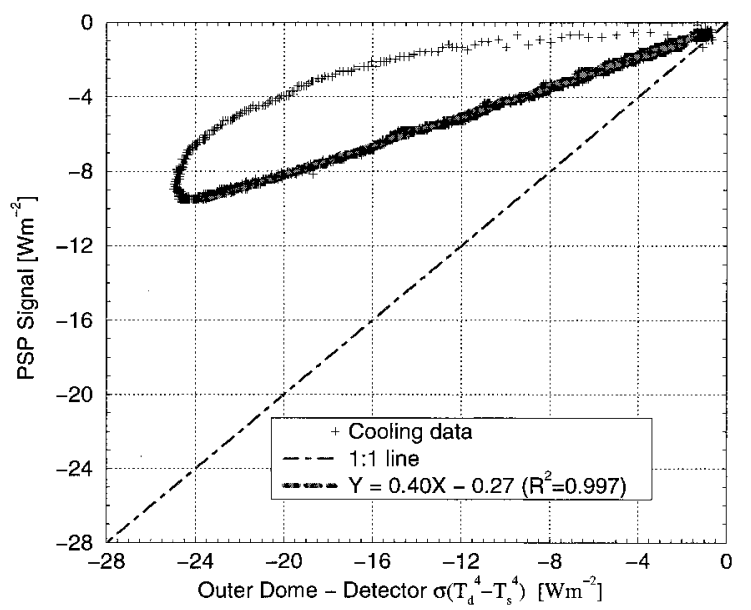

Fig. 6. Relationship between the outer-dome-to-detector theoretical blackbody radiative exchange and the PSP output signal during a cooling experiment.

inner dome. The inner dome itself is cooled mainly by radiative exchange with the outer dome. During the cooling experiment, the temperature decreases monotonically from the detector to the outer dome; the temperature gradient between the detector and the inner dome is of the order of $1^{\circ} \mathrm{C}$, whereas it can be $4{ }^{\circ} \mathrm{C}$ between the detector and the outer dome. By maintaining a temperature close to that of the detector, the inner dome effectively limits the radiative loss of the detector and hence limits the thermal offset. The slope and the intercept of the regression in Fig. 6 are 0.4 and $-0.3 \mathrm{~W} \mathrm{~m}^{-2}$, respectively. From a similar experiment, Bush et al. ${ }^{14}$ report a slope of 0.7 and an intercept of $-0.1 \mathrm{~W} \mathrm{~m}^{-2}$. In the experiment performed by Bush et al. the outer-dome thermistors were attached close to base of the dome, ${ }^{14}$ thus measuring a smaller temperature gradient than in our experiment, which explains the larger slope.

To illustrate the effect of the inner dome on the thermal offset, we perform a cooling experiment with a modified PSP that has no inner dome. Figure 7 shows the relationship between the outer-dome-todetector radiative exchange and the PSP output signal. The instrument offset reaches $-28 \mathrm{~W} \mathrm{~m}^{-2}$, which is significantly greater than the $-9 \mathrm{~W} \mathrm{~m}^{-2}$ obtained for similar cooling conditions with the twodome PSP. A linear regression is fitted through the data, which yields a slope of 1.1 and a $1-\mathrm{W} \mathrm{m}^{-2}$ intercept. As is noted in Subsection 2.A, the 2-mm outer glass dome has an IR emissivity of $\sim 0.9$ and an IR transmissivity close to 0 (nearly opaque). In this experiment the energy loss by the detector is almost completely due to radiative exchange between the detector and the 2-mm dome.

The cooling experiment is repeated with a PSP equipped with a 1-mm inner dome and no outer dome. We find a slope of 2.7 between the dome-todetector radiative exchange and the PSP output signal (not shown), which is close to the original slope of 2.38 with the two-dome instrument. The larger value of the slope is due to the energy lost by the

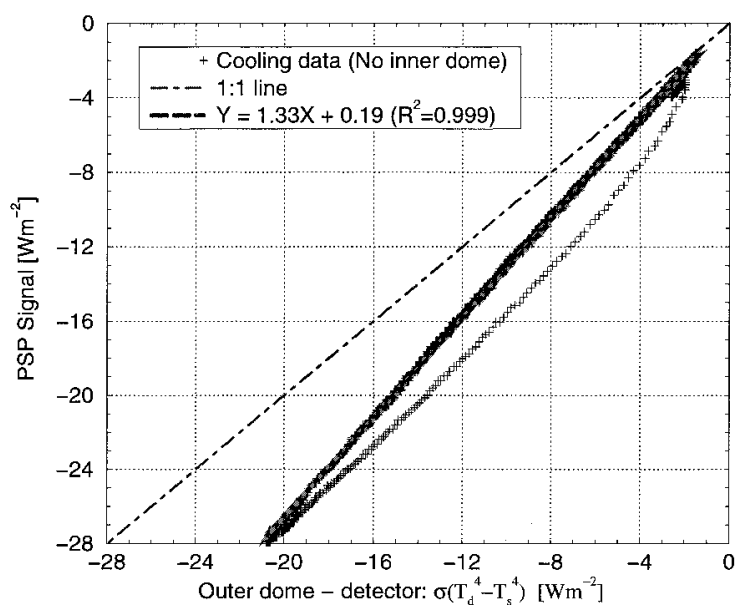

Fig. 7. Relationship between the outer-dome-to-detector theoretical blackbody radiative exchange and the PSP output signal during a cooling experiment, in which the inner dome of the PSP is removed.

detector to the inside walls of the refrigerator (by transmission through the 1-mm dome), which are colder than the 2-mm dome on the two-dome instrument.

These experiments illustrate the importance of using glass domes that are optically thick in the IR. According to a study by Gardon ${ }^{16}$ of the optical properties of glass (index of refraction $n=1.5$ ), the average transmissivity, spectrally weighted by the Planck function at $300 \mathrm{~K}$, of a glass sheet $1 \mathrm{~mm}$ or thinner can be significantly greater than 0 , whereas $2-\mathrm{mm}$ and thicker glass sheets can be considered optically thick for wavelengths greater than $5 \mu \mathrm{m}$. A 2-mmthick inner dome would be more efficient at limiting the thermal offset.

\section{B. Nighttime and Daytime Field Measurements}

A solar tracker, which can accommodate two instruments, is installed on the roof of a 15-m-tall building. The upward hemisphere is completely unobstructed from the roof. We mount a modified PSP and a standard Eppley PIR side by side, both with tracking shades. The two instruments are ventilated with standard Eppley ventilators. The PSP dome thermistor is facing away from the Sun at all times. The instruments are operated on a continuous basis at night and during the day for a period of 2 weeks in mid-November 1999.

We use a Campbell Model 21X datalogger to record the output of the PSP thermopile, the PSP body thermistor, the PSP dome thermistor, and the PIR thermopile output. The data are sampled every second. The nighttime data are extracted from the continuous time series. Figure 8 shows the relationship between the theoretical inner-dome-to-detector radiative exchange and the PSP thermopile output. A linear regression is fitted through the data, yielding a slope of 2.8 and an intercept of $-0.8 \mathrm{~W} \mathrm{~m}^{-2}$. The slope is different from that derived in the laboratory. The difference may be explained by the fact that the 


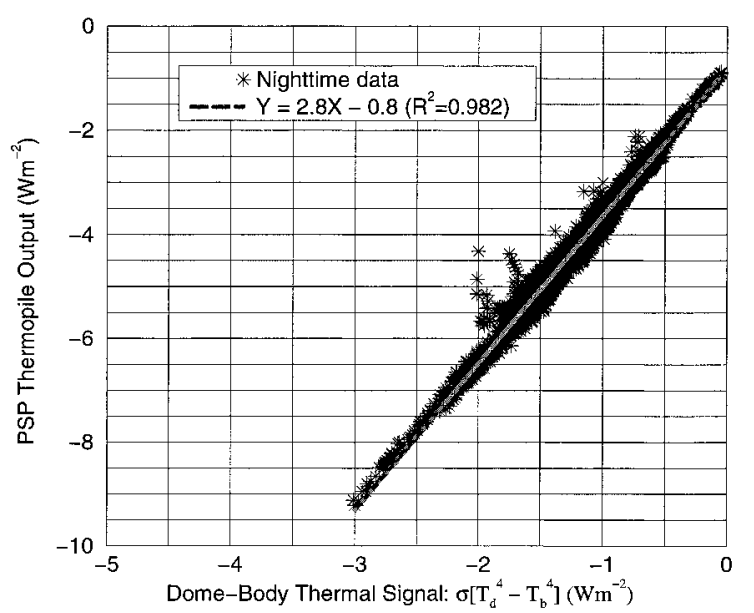

Fig. 8. Relationship between the inner-dome-to-detector theoretical blackbody radiative exchange and the PSP output signal during rooftop measurements at night (25 November to 2 December 1999).

slope is sensitive to the particular location where the dome temperature is measured. In the laboratory experiment the spatial temperature distribution on the dome was influenced by the nonuniform temperature distribution in the refrigerator. In our field experiment the temperature of the dome radiating to a clear sky is more uniform, especially since the instrument is ventilated. If the thermistor were moved closer to the tip of the dome, it would measure a colder temperature, which would yield a smaller slope in the linear regression. The magnitude of the intercept corresponds to a temperature gradient between the inner dome and the detector of $\sim 0.05{ }^{\circ} \mathrm{C}$. More than $98 \%$ of the variability in PSP output can be explained by the temperature gradient between the inner dome and the detector.

\section{Results and Discussion}

\section{A. Pyranometer Thermal Offset Determination from} Temperature Measurements

During daytime the PSP output signal contains both the shortwave component from solar radiation and the thermal offset that is due to the temperature gradient in the instrument. We use the PSP temperature measurements to estimate the PSP thermal offset. Once estimated, the thermal offset is removed from the PSP output and a corrected diffuse solar irradiance is produced.

Equation (5) is used to compute the PSP thermal offset based on PSP dome and body temperature measurements. Figure 9 shows $18 \mathrm{~h}$ of diffuse solar irradiance measurements recorded on 1 December 1999, a mostly clear day with a few high-altitude clouds in the morning and a northeasterly wind blowing between 5 and $10 \mathrm{~m} / \mathrm{s}$ with gusts up to $15 \mathrm{~m} / \mathrm{s}$. The nighttime surface temperature is close to $0{ }^{\circ} \mathrm{C}$, and the daytime temperature reaches $20^{\circ} \mathrm{C}$. The black solid curve represents 1-min averaged PSP output signal. The signal is quite variable with fre-

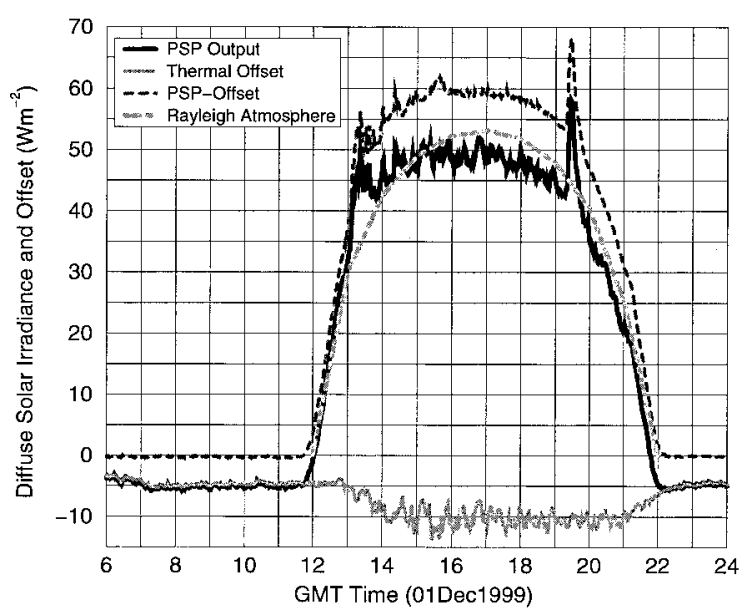

Fig. 9. Time series of PSP output signal (solid curve), computed diffuse solar irradiance at the surface in Rayleigh atmosphere (dotted-dashed curve), PSP thermal offset derived from temperature measurements (dotted curve), and corrected PSP output signal (dashed curve) under clear-sky conditions (1 December 1999).

quent $5-\mathrm{Wm}^{-2}$ jumps occurring over less than 5 min. This variability can be due to broken subvisual cirrus that indeed modify the diffuse solar irradiance or to thermal changes in the instrument, which are due to variable convective conditions that affect the thermal offset. The dotted-dashed curve shows the diffuse solar irradiance at the surface for a molecular atmosphere that is due solely to Rayleigh scattering, which represents the lower limit for diffuse radiation under clear skies. The Rayleigh limit is computed with a two-stream radiative transfer model. ${ }^{24,25}$ From 1500 GMT to sunset, the irradiance estimated by the PSP is below the molecular atmosphere level, which is not physical. The dotted curve at the bottom of Fig. 9 is the PSP thermal offset estimated from the PSP temperature measurements. At night the thermal offset is stable at approximately $-5 \mathrm{~W} \mathrm{~m}^{-2}$, whereas during daytime the offset is variable with a mean value close to $-10 \mathrm{~W} \mathrm{~m}^{-2}$. The daytime variability of the offset is significant; the offset can change by more than $5 \mathrm{~W} \mathrm{~m}^{-2}$ in less than $5 \mathrm{~min}$. Most of the fluctuations of the offset appear to be correlated with the high-frequency variations of the PSP output signal. When the magnitude of the offset decreases, the PSP output signal increases by the same amount. The high-frequency variability in thermal offset can be explained by turbulent convective heat exchange with the surrounding air that affects the temperature of the dome more rapidly than the temperature of the body. One might argue that the high-frequency variability in thermal offset could instead be due to spurious fluctuations in the dome temperature measurements associated with the absorption of solar radiation by the dome thermistor. However, the fluctuations of the diffuse solar irradiances and the thermal offsets are of the same magnitude, of the order of $5 \mathrm{~W} \mathrm{~m}^{-2}$. A decrease in thermal offset of $5 \mathrm{~W} \mathrm{~m}^{-2}$ can occur if the inner-dome temperature increases by $\sim 0.25^{\circ} \mathrm{C}$, assuming that 
the body temperature remains constant. From the capping experiments described in Subsection 4.B, we estimate that the diffuse solar irradiance would have to increase suddenly by more than $200 \mathrm{~W} \mathrm{~m}^{-2}$ for the temperature of the thermistor on the inner dome to increase by $0.25{ }^{\circ} \mathrm{C}$. We conclude that, during our daytime measurements, the variability in convective heat transfer between the dome and the surrounding air is responsible for the fluctuations in thermal offset and PSP output; in addition to the gusty wind conditions, significant surface heating during the day leads to vigorous turbulent convective heat transfer with the boundary layer. The thermal offset and PSP output are more stable at night because the convective heat transfer is reduced because of the nocturnal inversion in the column of air above the instrument.

The diurnal variation of the offset between nighttime and daytime under constant clear skies is due to the diurnal variation in the instrument temperature. At night, the instrument temperature is close to $0{ }^{\circ} \mathrm{C}$, whereas the daytime temperature reaches almost $20{ }^{\circ} \mathrm{C}$. So the gradient between the instrument temperature and the brightness temperature of the sky is less at night than during the day; hence the smaller offset at night. Note that the offset remains close to $-5 \mathrm{~W} \mathrm{~m}^{-2}$ for more than $1 \mathrm{~h}$ after sunrise, which occurs just before 1200 GMT. The instrument temperature starts to increase close to 1300 GMT when the diffuse solar irradiance has already reached $50 \%$ of the daily maximum value. When the instrument body starts to warm up, the efficient radiative cooling of the dome to the clear skies amplifies the temperature gradient and thus the thermal offset.

The dashed curve in Fig. 9 represents the corrected diffuse solar irradiance, defined as

$$
E^{\text {CorrDiff }}=E^{\text {Diff }}-E^{\text {Offset }}
$$

The corrected diffuse solar irradiances are $\sim 20 \%$ greater than the standard PSP irradiances, more than $5 \mathrm{~W} \mathrm{~m}^{-2}$ larger than the irradiances computed for a molecular atmosphere. The high-frequency variations, which are due to the turbulent surrounding air and observed in the uncorrected PSP output, are considerably reduced. The corrected nighttime values are close to $0 \mathrm{~W} \mathrm{~m}^{-2}$, as expected.

\section{B. Validation of Thermal Offset Estimates}

We can make an accurate estimate of the true thermal offset of the PSP during daytime operation by taking advantage of the quick time response of the thermopile detector. ${ }^{16}$ The time constant of the Eppley PSP thermopile is of the order of 1 or $2 \mathrm{~s}$, whereas the time constant of the PSP body-dome system is of the order of minutes. To estimate the true thermal offset at a time $t=t_{0}$, we perform the following experiment. At a time $t=t_{0}$, we cap the instrument with a white ceramic cup at ambient temperature, effectively shading the detector from all solar radiation, while the IR radiative exchanges between the detector and the domes remain. The ther-

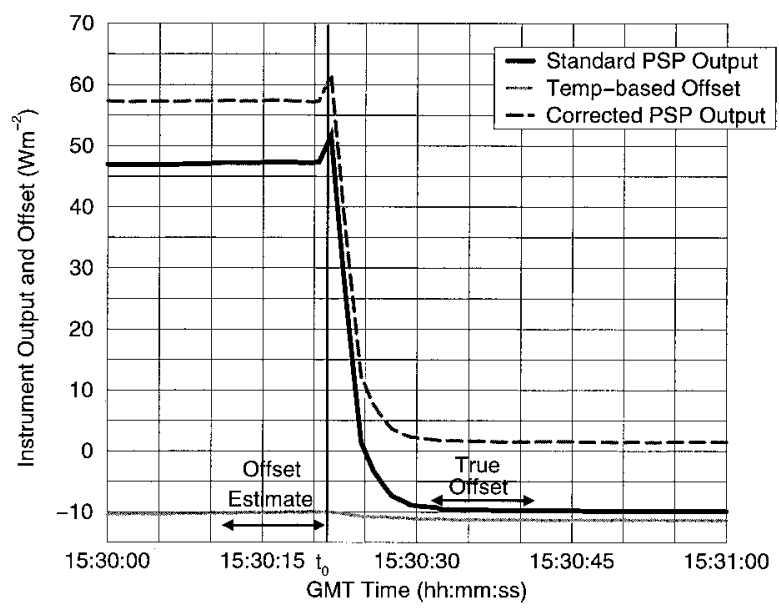

Fig. 10. Effect of instrument capping on the PSP output signal (solid curve) and the temperature-derived thermal offset (dotted curve). The corrected PSP output signal is shown as the dashed curve.

mopile responds immediately to the modified radiative input. The output of the thermopile is then driven only by the thermal radiative exchange between it and the domes, which can be considered constant for $30 \mathrm{~s}$, given the slower time response of the domes. A thermopile with a 1-2-s time constant subjected to a step input reaches $99.99 \%$ of the steady-state value within $10-20 \mathrm{~s}$. Hence we define the true thermal offset at time $t=t_{0}$ to be the $10-\mathrm{s}$ average of the signal produced by the thermopile from $t=t_{0}+10 \mathrm{~s}$ to $t=t_{0}+20 \mathrm{~s}$. We also define our estimate of the thermal offset at time $t=t_{0}$ to be the 10 -s average of the offset estimated from the instrument temperatures from $t=t_{0}-10 \mathrm{~s}$ to $t=t_{0}$. The 10 -s averages allow us to remove some of the noise in the data.

Figure 10 shows an example of a 1-s instrument output during a capping experiment. At 15:30:00 GMT the shaded PSP measures the diffuse solar irradiance under clear-sky conditions. At 15:30:21 GMT $\left(t_{0}\right)$ the instrument is capped and the instrument output shown as the black solid curve drops very rapidly. The true offset given as the average instrument output between 15:30:31 and 15:30:41 GMT is $-9.7 \mathrm{~W} \mathrm{~m}^{-2}$, and the estimated offset given by the value of the dotted curve between 15:30:21 and 15:30:31 GMT is $-10.1 \mathrm{~W} \mathrm{~m}^{-2}$. The corrected instrument output defined as the standard PSP output minus the estimated offset is shown as the dashed curve. The corrected output drops within $1 \mathrm{~W} \mathrm{~m}^{-2}$ of zero $\sim 10 \mathrm{~s}$ after the instrument is capped.

We performed 14 independent capping experiments at different times of the day and under various cloud-cover conditions. Figure 11 is a scatter diagram of the true thermal offsets from the capped instrument signal versus the offsets estimated with the instrument temperature measurements. The 14 data points line up closely to the 1:1 line. The mean difference between the estimates and the true offsets is $0.5 \mathrm{~W} \mathrm{~m}^{-2}$, with a $95 \%$ confidence interval of \pm 0.2 


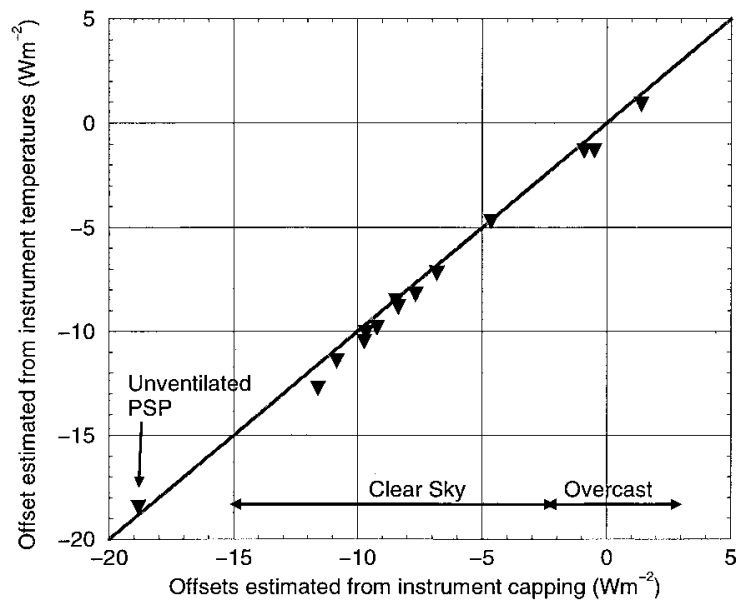

Fig. 11. True thermal offsets estimated from capping experiments versus thermal offsets derived from temperature measurements.

$\mathrm{W} \mathrm{m}{ }^{-2}$. These data show that we can estimate the daytime thermal offset of the PSP to better than $1 \mathrm{~W}$ $\mathrm{m}^{-2}$. For a shaded and ventilated PSP operated in December at our NASA Langley site, the offset is in the $\pm 5-\mathrm{W} \mathrm{m} \mathrm{m}^{-2}$ range under overcast skies, and it ranges between -5 and $-15 \mathrm{~W} \mathrm{~m}^{-2}$ under clear skies. For an unventilated PSP under clear skies, the offset reached $-20 \mathrm{~W} \mathrm{~m}^{-2}$.

\section{Pyranometer Thermal Offset Determination from Pyrgeometer Measurements}

Several authors ${ }^{26-28}$ have suggested that the PIR instrument could be used as a means to predict the PSP offset. The radiative cooling of an up-looking PSP to a clear sky or to high-altitude clouds results in a temperature gradient in the instrument that produces a thermal offset signal. These conditions correspond to the typical negative net (downwelling minus upwelling) IR irradiance at the surface. The output of a PIR instrument is proportional to the net-IR irradiance on its detector; the silicon dome of the PIR absorbs and reflects the incoming solar radiation. The net-IR irradiance on the detector is composed of a downwelling IR component from the sky that is transmitted through the dome, a downwelling component emitted by the dome, and an upwelling component emitted by the detector itself. Similarly, the output of the PSP that is due to thermal gradients is proportional to the net-IR irradiance on its detector. The assumption of Refs. 26-28 is that the PSP thermal offset can be correlated to the PIR detector output. Figure 12 shows the relationship between the PSP thermopile output and the PIR thermopile output at night, based on ten nights of data collected at our NASA Langley site in December 1999 (described in Subsection 3.B). The data reveal that the negative thermal offset of the PSP increases as the negative surface net-IR irradiance increases. The data can be fitted with a linear regression of the form

$$
\text { Offset }^{\mathrm{PSP}}=A \times \mathrm{PIR}+B \text {. }
$$

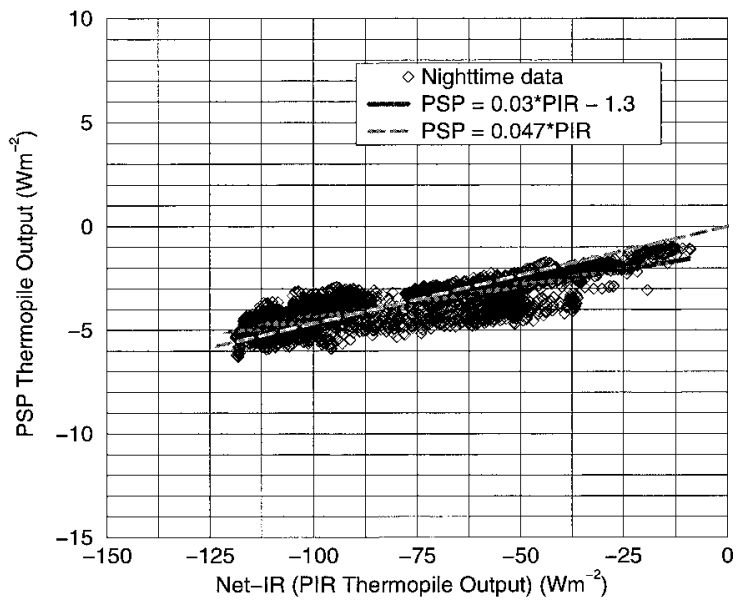

Fig. 12. Relationship between the net-IR signal from a PIR and the PSP output signal during rooftop measurements at night (25 November to 2 December 1999). The solid curve shows the unmodified regression (slope 0.03), and the dashed curve shows the regression forced through a zero intercept (slope 0.047).

The slope is 0.03 , which means that the sensitivity of the PSP offset to net IR is quite small. The intercept is of the order of $-1 \mathrm{~W} \mathrm{~m}^{-2}$, which is similar to the intercept obtained in Fig. 8. Alberta and Charlock ${ }^{27}$ suggest that the regression should be rotated around the centroid of the data to force it through the origin $(0,0)$, because a net IR of zero should correspond to a PSP offset of zero. In this case the slope becomes 0.047 , which is a value comparable with that reported by other authors. ${ }^{26,27}$

During daytime the PSP output signal contains both a shortwave component from solar radiation and a thermal offset that is due to the temperature gradients in the instrument. The PSP thermal offset is estimated from the PIR output signal by use of Eq. (7). Once estimated, the thermal offset is removed from the standard PSP output and a corrected solar irradiance is produced.

Equation (7) is used to compute the PSP thermal offset based on the PIR output signal; coefficients $A=$ 0.047 and $B=0$ are used for consistency with Refs. 27 and 28 . Figure 13 shows $18 \mathrm{~h}$ of PSP and PIR measurements for the same clear and windy day as that in Fig. 9. The black solid curve represents 1-min averaged shaded PSP output signal. The dotted-dashed curve shows the diffuse solar irradiance at the surface for a molecular atmosphere that is due solely to Rayleigh scattering, which represents the lower limit for diffuse radiation under clear skies. From 1500 GMT to sunset the irradiance estimated by the PSP is below the molecular atmosphere level. The dotted curve at the bottom of Fig. 13 is the PSP thermal offset estimate based on the PIR measurement. This estimate is very stable with little diurnal variation $\left(-5 \mathrm{~W} \mathrm{~m}^{-2}\right.$ at night and a peak at $-7 \mathrm{~W}$ $\mathrm{m}^{-2}$ during the day). The dashed curve is the estimate of the diffuse solar irradiance based on the PSP output minus the PIR-derived thermal offset. At night the irradiance estimates are stable at $0 \mathrm{~W} \mathrm{~m}^{-2}$, 


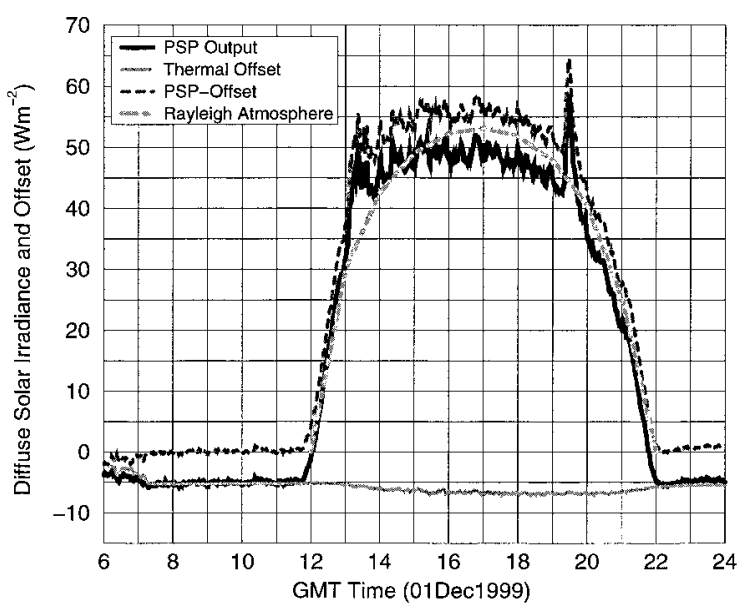

Fig. 13. Time series of PSP output signal (solid curve), computed diffuse solar irradiance at the surface in Rayleigh atmosphere (dotted-dashed curve), PSP thermal offset derived from net-IR measurements (dotted curve), and corrected PSP output signal (dashed curve) under clear-sky conditions (1 December 1999).

which is consistent with the linear regressions of Fig. 12. During the day the diffuse solar irradiance estimate is at or above the physical minimum of the molecular atmosphere; the thermal offset derived from the PIR output here underestimates the true offset by $25 \%$.

\section{Variability of the Pyranometer Thermal Offset}

Figure 14 shows eight panels representing the diurnal cycles of diffuse solar irradiance for eight consecutive days (25 November to 2 December 1999, days of the year 329-336) measured at the NASA Langley rooftop site. Each panel shows three curves: black curve, the irradiance from a standard PSP; red curve, a corrected irradiance (irradiance - offset) calculated from our temperature-based estimate of the PSP offset; and green curve, a corrected irradiance (irradiance offset) calculated from the PIR-based estimate of the PSP offset. Days 329 and 330 are mostly cloudy, and the diffuse irradiances range between 100 and $300 \mathrm{~W} \mathrm{~m}^{-2}$, whereas the remainder of the period is mostly clear with irradiances below 100 $\mathrm{W} \mathrm{m}^{-2}$. On mostly clear days the irradiances corrected with PSP temperatures are larger than the standard irradiances by $10 \%$ to $20 \%$; the irradiances corrected with the PIR signal are $10 \%$ to $15 \%$ larger than the standard PSP irradiances. For cloudy days the diffuse irradiances are generally much larger, and the differences between corrected and uncorrected PSP outputs are less than 2\%. At night the temperature-based corrected irradiance is consistently zero. The PIR-based corrected irradiance is close to zero as well, except for day 331.

The eight panels of Fig. 15 represent the PSP offset estimates based on the methods described in Subsections 4.A and 4.B for the same days as those in Fig. 14. The offsets derived from temperature measurements are shown in red, and the offsets based on the PIR measurements are shown in green. The PIRbased offset estimates are based on the regression with the larger slope (slope $=0.047$ and intercept $=$ 0; see Fig. 11). The largest offsets are observed during daytime under clear-sky conditions with values ranging from -5 to $-15 \mathrm{~W} \mathrm{~m}^{-2}$. On day 329 , broken low-altitude clouds $(75 \%$ cloud cover and $280 \mathrm{~K}$ brightness temperature) move across the area, re-
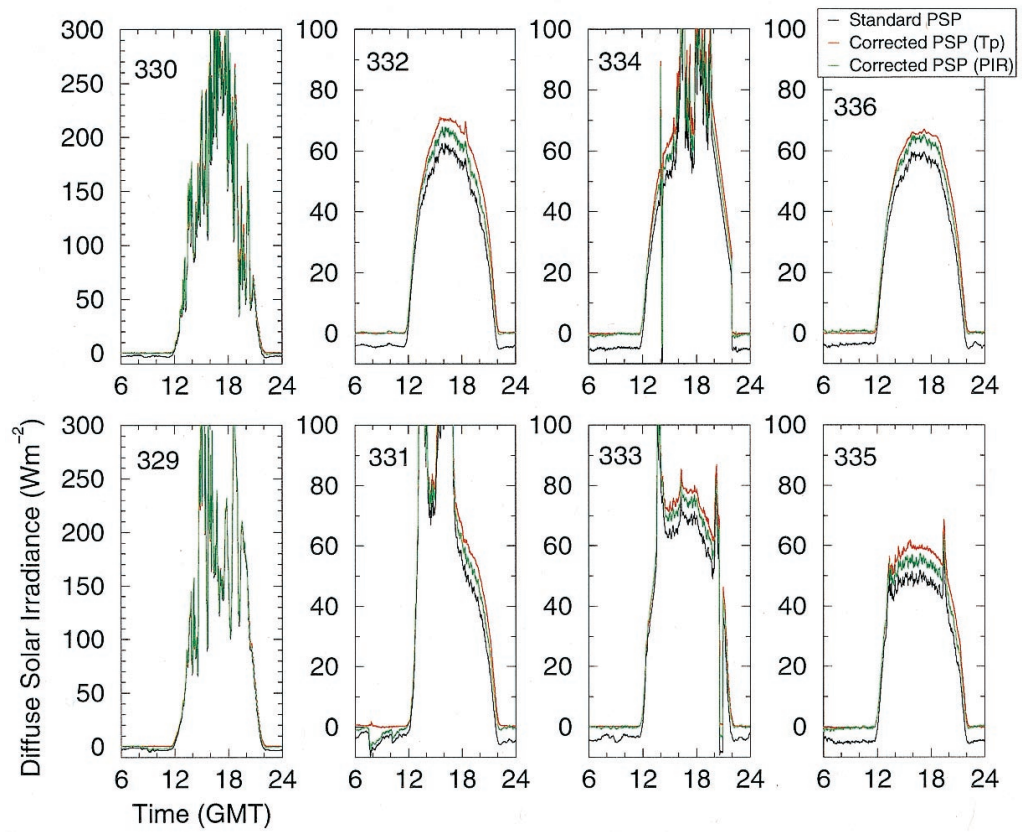

Fig. 14. Eight diurnal cycles of PSP output signals during daytime and nighttime rooftop measurements (25 November to 2 December 1999, days of the year 329-336). The standard PSP output signal is shown in black, the corrected PSP with PSP temperatures in red, and the corrected PSP with net-IR in green. Days 329 and 330 are mostly cloudy, and days 331-336 are mostly clear. 

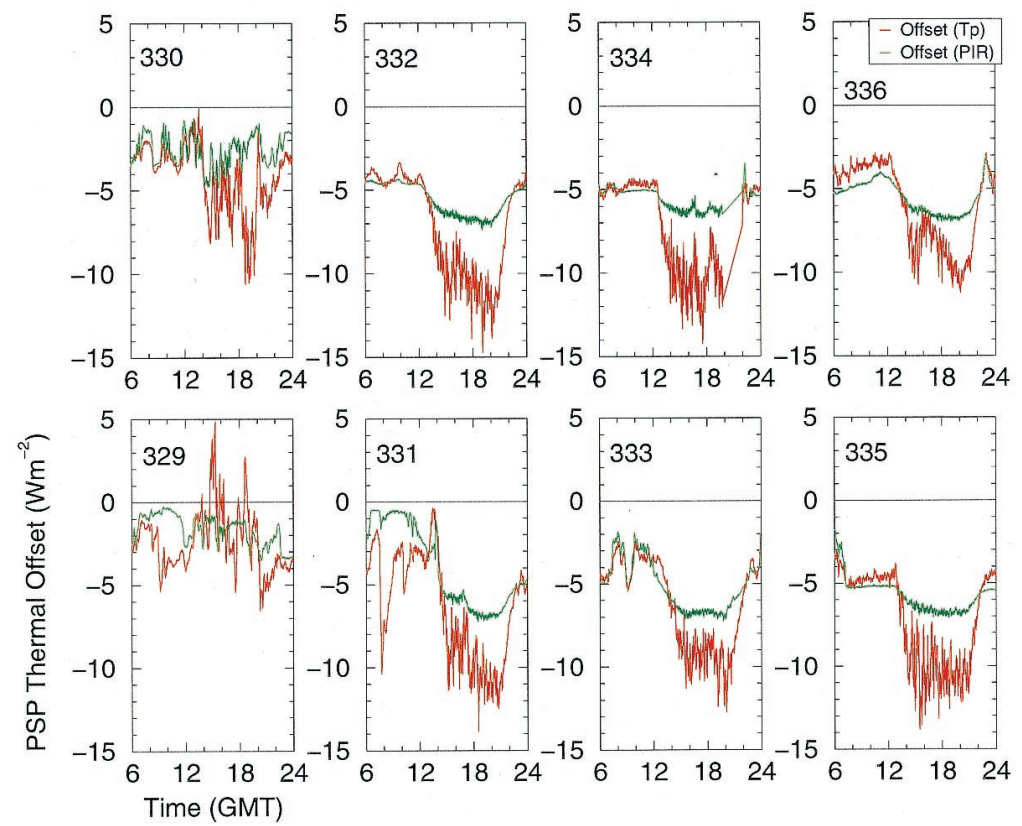

Fig. 15. Eight diurnal cycles of estimated PSP offsets during daytime and nighttime rooftop measurements (25 November to 2 December 1999, days of the year 329-336). The temperature-derived offset is shown in red, and the offset derived from net-IR measurements is shown in green. Days 329 and 330 are mostly cloudy, and days 331-336 are mostly clear.

sulting in daytime offsets close to zero on average, but ranging between -5 and $+5 \mathrm{~W} \mathrm{~m}^{-2}$. On day 330 , the low clouds are scattered (25\% cloud cover), which leads to offsets ranging from -5 to $-10 \mathrm{~W} \mathrm{~m}^{-2}$.

Figure 15 reveals that the nighttime estimates of the offsets of the two methods agree well, whereas they differ significantly during daytime under clear skies. This is an indication that the relationship between the PSP offset and the net-IR signal from the PIR changes between day and night. In addition, the high-frequency variability of the daytime PSP offset (described in Subsection 4.A) is not picked up by the PIR-based technique. Therefore the highfrequency variations remain in the corrected PSP output based on the PIR technique, but the highfrequency variations of the corrected PSP output based on the PSP temperature technique are considerably reduced. This is most apparent on days 332 , 335, and 336 in Fig. 15.

The daytime thermal offsets reported by Bush et $a l .,{ }^{14}$ derived from dome and body temperature measurements, range from -16 to $-23 \mathrm{~W} \mathrm{~m}^{-2}$ for a shaded PSP under clear-sky conditions. These offsets are significantly greater than the ones reported in the present paper because their instruments were not ventilated. In addition, their experiment took place in La Jolla, California, which is a drier climate than Hampton, Virginia, thus permitting greater IR cooling of the domes.

\section{Conclusions}

When the dome temperature is less than the temperature of the cold junction of the thermopile, the pyranometer underestimates the solar irradiance because of the net energy loss by the detector through IR radiation with the dome. The dome temperature is usually less than the cold junction temperature under clear-sky conditions because (1) the dome faces the sky, whose brightness temperature is typically at least $30^{\circ} \mathrm{C}$ less than the cold junction temperature; (2) the dome has a low thermal conductivity and heat capacity; (3) the emissivity of the dome integrated over the emission spectrum of the dome under operating conditions is 0.9 ( 0.8 for the 1 -mm inner dome); and (4) the dome is nearly transparent to solar radiation, whereas the detector absorbs solar radiation.

The magnitude of the offset depends on meteorological conditions such as brightness temperature of the sky, surface temperature, and wind speed. It also depends on the design of the pyranometer; the smaller the IR transmissivity of the domes integrated over the emission spectrum of the sky, the smaller the offset. Importantly, this offset cannot be eliminated in the pyranometer calibration process that is widely adopted by the community (e.g., the Baseline Surface Radiation Network).

In this paper we show that the offset in diffuse solar irradiance measurements can be computed from the temperature of the inner dome and the detector cold junction by use of a simple relationship relating the temperature difference to the offset. The daytime offsets observed on our ventilated and shaded PSP operating at our NASA Langley rooftop site during clear days of December 1999 range from -5 to $-15 \mathrm{~W} \mathrm{~m}^{-2}$.

The offsets during daytime are usually greater than during nighttime because the cold junction temperature is greater during daytime, following the diurnal cycle of the surface temperature. Because of 
this diurnal cycle, the empirical estimate of the offset based on nighttime PIR data tends to underestimate the value and the high-frequency variations of the daytime offset of the PSP, whereas our technique based on PSP temperatures estimates the offset of a shaded PSP to better than $1 \mathrm{~W} \mathrm{~m}^{-2}$.

\section{References}

1. L. J. B. McArthur, Baseline Surface Radiation Network (BSRN) operations manual, Version 1.0, WMO/TD 879 (World Meteorological Organization, Geneva, 1998).

2. Guide to meteorological instruments and methods of observation, WMO 8, 6th ed. (Secretariat of the World Meteorological Organization, Geneva, 1996).

3. I. R. Reda, "Improving the accuracy of using pyranometers to measure the clear sky global solar irradiance," in Proceedings of the Ninth ARM Science Team Meeting (available from the U.S. Department of Commerce, Springfield, Va. 22161, 1999).

4. J. Michalsky, E. Dutton, M. Rubes, D. Nelson, T. Stoffel, M. Wesley, M. Splitt, and J. DeLuisi, "Optimal measurement of surface shortwave irradiance using current instrumentation," J. Atmos. Oceanic Technol. 16, 55-69 (1999).

5. A. J. Drummond and J. J. Roche, "Corrections to be applied to measurements made with Eppley (and other) spectral radiometers when used with Schott colored glass filters," J. Appl. Meteorol. 4, 741-744 (1965).

6. N. Robinson, Solar Radiation (Elsevier, New York, 1966), pp. 247-271.

7. A. Gulbrandsen, "On the use of pyranometers in the study of spectral solar radiation and atmospheric aerosols," J. Appl. Meteorol. 17, 899-904 (1978).

8. T. P. Charlock and T. L. Alberta, "The CERES/ARM/GEWEX experiment (CAGEX) for the retrieval of radiative fluxes with satellite data," Bull. Am. Meteorol. Soc. 77, 2673-2683 (1996).

9. S. Kato, T. P. Ackerman, E. E. Clothiaux, J. H. Mather, G. G. Mace, M. L. Wesely, F. Murcray, and J. Michalsky, "Uncertainties in modeled and measured clear-sky surface shortwave irradiances," J. Geophys. Res. 102, 25881-25898 (1997).

10. S. Kato, T. P. Ackerman, E. G. Dutton, N. Laulainen, and N. Larson, "A comparison of modeled and measured surface shortwave irradiance for a molecular atmosphere," J. Quant. Spectrosc. Radiat. Transfer 61, 493-502 (1999).

11. R. D. Cess, Institute for Planetary Atmospheres, Marine Sciences Research Center, State University of New York, Stony Brook, N.Y. 11794-5000 (personal communication, 1999).

12. M. Wild, "Discrepancies between model-calculated and observed shortwave atmospheric absorption in areas with high aerosol loadings," J. Geophys. Res. 104, 27361-27373 (1999).

13. W. C. Conant, "An observational approach for determining aerosol surface radiative forcing: results from the first field phase of INDOEX," J. Geophys. Res. 105, 15347-15360 (2000).

14. B. C. Bush, F. P. J. Valero, A. S. Simpson, and L. Bignone, "Characterization of thermal effects in pyranometers: a data correction algorithm for improved measurement of surface insolation," J. Atmos. Oceanic Technol. 17, 165-175 (2000).

15. J. Hickey, The Eppley Laboratory, Inc., P.O. Box 419, 12 Sheffield Ave., Newport, R.I. 02840 (personal communication, 1999).

16. R. Gardon, "The emissivity of transparent materials," J. Am. Ceramic Soc. 39, 278-285 (1956).

17. M. F. Modest, Radiative Heat Transfer (McGraw-Hill, New York, 1993).

18. B. W. Forgan, "A new method for calibrating reference and field pyranometers," J. Atmos. Oceanic Technol. 13, 638-645 (1996).

19. B. Albrecht, M. Peollet, and S. K. Cox, "Pyrgeometer measurements from aircraft," Rev. Sci. Instrum. 45, 33-38 (1974).

20. R. Philipona, C. Frohlich, and Ch. Betz, "Characterization of pyrgeometers and the accuracy of atmospheric long-wave radiation measurements," Appl. Opt. 34, 1598-1605 (1995).

21. C. W. Fairall, P. O. G. Persson, E. F. Bradley, R. E. Payne, and S. P. Anderson, "A new look at calibration and use of Eppley precision infrared radiometers. Part I: theory and applications," J. Atmos. Oceanic Technol. 15, 1229-1242 (1998).

22. M. P. Haeffelin, C. K. Rutledge, S. Kato, A. M. Smith, and J. R. Mahan, "The uncertainty in surface shortwave radiation measurements: experimental tests and numerical simulations of pyranometers," in Proceedings of the Ninth ARM Science Team Meeting (available from the U.S. Department of Commerce, Springfield, Va. 22161, 1999).

23. A. M. Smith, M. P. Haeffelin, F. J. Nevarez, J. R. Mahan, S. Kato, and C. K. Rutledge, "Experimental and theoretical study of uncertainty in pyranometers for surface radiation," in Sensors, Systems, and Next-Generation Satellites III, H. Fujisada and J. Lurie, eds., Proc. SPIE 3870, 536-547 (1999).

24. O. B. Toon, C. P. Mckay, and T. P. Ackerman, "Rapid calculation of radiative heating rates and photodissociation rates in inhomogeneous multiple scattering atmosphere," J. Geophys. Res. 94, 16287-16301 (1989).

25. J. E. Hansen and L. D. Travis, "Light scattering in planetary atmospheres," Space Sci. Rev. 16, 527-610 (1974).

26. E. G. Dutton, Climate Monitoring and Diagnostics Laboratory, National Oceanic and Atmospheric Administration R/CMDL1, 325 Broadway, Boulder, Colo. 80303 (personal communication, 2000).

27. T. L. Alberta and T. P. Charlock, "A comprehensive resource for the investigation of shortwave fluxes in clear conditions: CAGEX version 3," in Preprints of the Tenth Conference on Atmospheric Radiation (American Meteorological Society, Boston, Mass., 1999), pp. 279-282.

28. R. N. Halthore, S. E. Schwartz, and E. G. Dutton, "Diffuse shortwave irradiance at surface-further issues and implications," in Proceedings of the Ninth ARM Science Team Meeting (available from the U.S. Department of Commerce, Springfield, Va. 22161, 1999). 